\title{
Clinical trial data validate the C-peptide estimate model in type 1 diabetes
}

\author{
John M. Wentworth ${ }^{1,2,3}$ (D) Naiara G. Bediaga ${ }^{3} \cdot$ Stephen E. Gitelman ${ }^{4} \cdot$ Carmela Evans-Molina $^{5}$ • Peter A. Gottlieb ${ }^{6}$. \\ Peter G. Colman ${ }^{1,2} \cdot$ Michael J. Haller $^{7}$. Leonard C. Harrison ${ }^{3}$
}

Received: 7 November 2019 / Accepted: 17 December 2019 / Published online: 26 January 2020

(C) Springer-Verlag GmbH Germany, part of Springer Nature 2020

\author{
Abbreviations \\ ATG Anti-thymocyte globulin \\ $\mathrm{CP}_{\mathrm{EST}} \quad$ Estimated $\mathrm{CP}_{\mathrm{STIM}}$ \\ $\mathrm{CP}_{\text {STIM }}$ Meal-stimulated C-peptide \\ G-CSF Pegylated granulocyte colony stimulating factor \\ IDAA1c Insulin dose-adjusted $\mathrm{HbA}_{1 \mathrm{c}}$
}

To the Editor: The area under the curve of meal-stimulated Cpeptide $\left(\mathrm{CP}_{\mathrm{STIM}}\right)$ is most commonly used as the primary outcome for clinical trials of immune therapy in recent-onset stage 3 type 1 diabetes [1]. However, because $\mathrm{CP}_{\text {STIM }}$ requires repeated venous blood sampling over 2 to $4 \mathrm{~h}$, it is burdensome to participants and its laboratory analysis is costly. Moreover, it is not convenient in the routine clinical setting, where interest in assessing beta cell function continues to grow given recent advances in immune therapy for type 1 diabetes $[2,3]$.

John M. Wentworth

wentworth@wehi.edu.au

1 Royal Melbourne Hospital Department of Diabetes and Endocrinology, Parkville, VIC, Australia

2 Royal Melbourne Hospital Department of Medicine, University of Melbourne, Parkville, VIC, Australia

3 Department of Population Health and Immunity, Walter and Eliza Hall Institute of Medical Research, 1G Royal Parade, Parkville, VIC 3052, Australia

4 Pediatric Diabetes, University of California at San Francisco, San Francisco, CA, USA

5 Center for Diabetes and Metabolic Diseases, Indiana University School of Medicine, Indianapolis, IN, USA

6 Barbara Davis Center for Childhood Diabetes, University of Colorado, Aurora, CO, USA

7 Pediatric Endocrinology, University of Florida, Gainesville, FL, USA
To simplify assessment of beta cell function, we developed a formula, ' $\mathrm{CP}_{\mathrm{EST}}$ ', that estimates $\mathrm{CP}_{\mathrm{STIM}}$ using six single-timepoint measures: disease duration, insulin dose, $\mathrm{BMI}, \mathrm{HbA}_{1 \mathrm{c}}$, fasting plasma glucose and fasting plasma C-peptide [4]. In the original publication, $\mathrm{CP}_{\mathrm{EST}}$ reliably identified treatment effects in three trials of immune therapy in recent-onset type 1 diabetes, suggesting it could be used as a simpler, less burdensome primary outcome measure. However, half of the data in these analyses had been used to develop the $\mathrm{CP}_{\mathrm{EST}}$ model and the model needed to be tested further by applying it to new data.

The recent availability of data from the TrialNet TN19 antithymocyte globulin (ATG) and pegylated granulocyte colony stimulating factor (G-CSF) trial (NCT02215200) [2] allows definitive testing of the utility of $\mathrm{CP}_{\mathrm{EST}}$ as a clinical trial outcome measure. TN19 compared, in children and young adults (age range 12 to 43 years) with recent-onset type 1 diabetes, the effects of ATG (2.5 mg/kg i.v. over 2 days) followed by G-CSF (6 mg s.c. fortnightly for 6 doses), ATG followed by placebo, and placebo followed by placebo. The primary endpoint was $\mathrm{CP}_{\mathrm{STIM}}$ at one year, assessed using an ANCOVA model adjusted for baseline age, baseline $\log _{e}\left(\mathrm{CP}_{\mathrm{STIM}}+1\right)$ and sex [2].

TN19 data were supplied with participant age rounded to the nearest year. We excluded two participants with incomplete data, resulting in 28, 29 and 30 participants in the ATG/G-CSF, ATG/ placebo and placebo/placebo groups, respectively. Statistical analyses were performed using R software (www.r-project.org).

Based on 432 measurements obtained from the 87 trial participants over the first year, $\mathrm{CP}_{\mathrm{STIM}}$ correlated strongly with

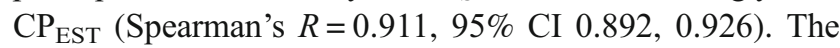
correlation between $\mathrm{CP}_{\mathrm{STIM}}$ and insulin dose-adjusted $\mathrm{HbA}_{1 \mathrm{c}}$ (IDAA1c), another proposed single-time-point measure based on $\mathrm{HbA}_{1 \mathrm{c}}$ and insulin dose [5], was significantly weaker (Spearman's $R-0.555,95 \% \mathrm{CI}-0.619,-0.484$ ).

Figure 1 presents the ATG/G-CSF trial primary outcome according to measured $\left(\mathrm{CP}_{\mathrm{STIM}}\right)$ and modelled $\left(\mathrm{CP}_{\mathrm{EST}}\right)$ beta cell function. Overall, the values and trajectory of each treatment group using $\mathrm{CP}_{\mathrm{STIM}}$ and $\mathrm{CP}_{\mathrm{EST}}$ were similar. The $p$ values for the month 12 outcomes for placebo/placebo vs ATG/ 


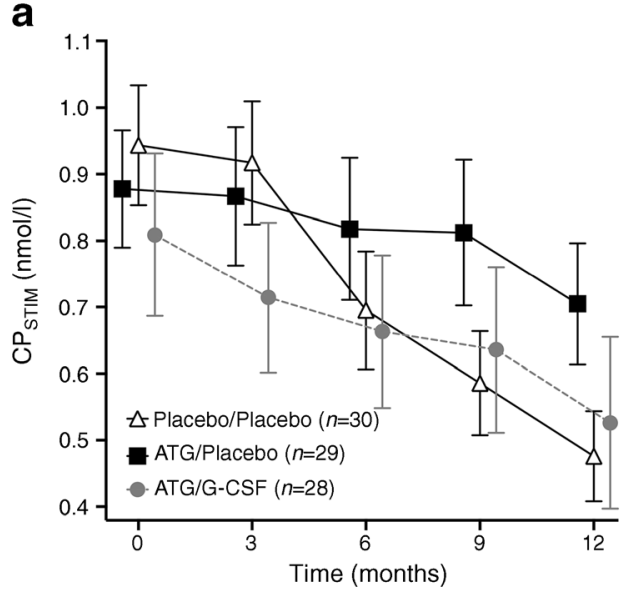

Fig. 1 TN19 outcomes according to (a) measured $\left(\mathrm{CP}_{\mathrm{STIM}}\right)$ and (b) modelled $\left(\mathrm{CP}_{\mathrm{EST}}\right)$ beta cell function. Data presented are mean $\pm \mathrm{SEM}$. Measurements were taken at the same time points but have been offset to prevent overlap and enable better comparisons of the groups. Group comparisons were performed using an ANCOVA model adjusted for

placebo and placebo/placebo vs ATG/G-CSF were 0.0007 and 0.0851 , respectively, for $\mathrm{CP}_{\mathrm{STIM}}$, and 0.0034 and 0.376 , respectively, for $\mathrm{CP}_{\mathrm{EST}}$; the $p$ values for IDAA1c (not shown in the figure) were 0.021 and 0.105 , respectively.

These findings provide further evidence that $\mathrm{CP}_{\mathrm{EST}}$ is a reasonable substitute for $\mathrm{CP}_{\text {STIM }}$ and is more accurate than IDAA1c for approximating beta cell function using singletime-point measures. The ability of $\mathrm{CP}_{\mathrm{EST}}$ to accurately identify treatment effects in a fully independent dataset supports the notion that it could be used as a primary outcome measure in future clinical trials in recent-onset stage 3 type 1 diabetes. Perhaps more compelling may be the suggestion that it could be used as a simple measure that incorporates readily available clinical and demographic information and fasting laboratory data to monitor the response to immune therapies when they are approved for clinical use.

Acknowledgements We acknowledge the support of the Type 1 Diabetes TrialNet Study Group, which identified study participants and provided samples and follow-up data for this study.

Data availability Data used for this study can be obtained through TrialNet (www.trialnet.org).

Funding The Type 1 Diabetes TrialNet Study Group is a clinical trials network funded by the National Institutes of Health (NIH) through the National Institute of Diabetes and Digestive and Kidney Diseases, the National Institute of Allergy and Infectious Diseases, and the Eunice Kennedy Shriver National Institute of Child Health and Human Development, through cooperative agreements U01 DK061010, U01 DK061034, U01 DK061042, U01 DK061058, U01 DK085465, U01 DK085453, U01 DK085461, U01 DK085466, U01 DK085499, U01 DK085504, U01 DK085509, U01 DK103180, U01 DK103153, U01 DK085476, U01 DK103266, U01 DK103282, U01 DK106984, U01 DK106994, U01 DK107013, U01 DK107014, UC4 DK106993, UC4 DK11700901 and the JDRF. The contents of this article are solely the

\section{b}

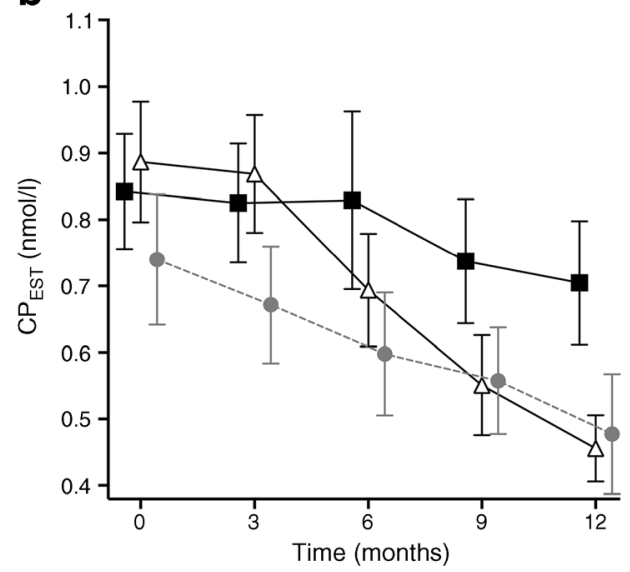

baseline age, baseline $\log _{e}\left(\mathrm{CP}_{\mathrm{STIM}}+1\right)$ and sex. The $p$ values for the month 12 outcomes for placebo/placebo vs ATG/placebo and placebo/ placebo vs ATG/G-CSF were 0.0007 and 0.0851 , respectively, for $\mathrm{CP}_{\mathrm{STIM}}$, and 0.0034 and 0.376 , respectively, for $\mathrm{CP}_{\mathrm{EST}}$

responsibility of the authors and do not necessarily represent the official views of the NIH or the JDRF.

Duality of interest The authors declare that there is no duality of interest associated with this manuscript.

Contribution statement All authors contributed to the conception and design of the study. MJH oversaw data collection for the TN19 study. JMW and NGB performed data analyses and interpretation. JMW drafted the manuscript, which all authors edited. Each author approved the submitted manuscript. JW is the guarantor of this work and takes full responsibility for the work as a whole, including the study design, access to data and the decision to submit and publish the manuscript.

\section{References}

1. Greenbaum CJ, Mandrup-Poulsen T, McGee PF et al (2008) Mixedmeal tolerance test versus glucagon stimulation test for the assessment of beta-cell function in therapeutic trials in type 1 diabetes. Diabetes Care 31(10):1966-1971. https://doi.org/10.2337/dc07-2451

2. Haller MJ, Schatz DA, Skyler JS et al (2018) Low-dose anti-thymocyte globulin (ATG) preserves $\beta$-cell function and improves $\mathrm{HbA}_{1 \mathrm{c}}$ in new-onset type 1 diabetes. Diabetes Care 41(9):1917-1925. https://doi.org/10.2337/dc18-0494

3. Herold KC, Bundy BN, Long SA et al (2019) An anti-CD3 antibody, teplizumab, in relatives at risk for type 1 diabetes. N Engl J Med 381(7):603-613. https://doi.org/10.1056/NEJMoa1902226

4. Wentworth JM, Bediaga NG, Giles LC et al (2019) Beta cell function in type 1 diabetes determined from clinical and fasting biochemical variables. Diabetologia 62(1):33-40. https://doi.org/10.1007/s00125-018-4722-z

5. Max Andersen ML, Hougaard P, Porksen S et al (2014) Partial remission definition: validation based on the insulin dose-adjusted HbA1c (IDAA1C) in 129 Danish children with new-onset type 1 diabetes. Pediatr Diabetes 15(7): 469-476. https://doi.org/10.1111/pedi.12208

Publisher's note Springer Nature remains neutral with regard to jurisdictional claims in published maps and institutional affiliations. 